\title{
Window Analysis Using Two-stage DEA in Heart Hospitals
}

\author{
Mirpouya Mirmozaffari ${ }^{1}$, and Alireza Alinezhad ${ }^{2}$ \\ ${ }^{1}$ Msc. Student, Department of Industrial Engineering, Faculty of Industrial and Mechanical Engineering, Qazvin \\ Branch, Islamic Azad University, Qazvin, Iran (Email:m.mirmozaffari@gmail.com) \\ ${ }^{2}$ Associate Professor, Department of Industrial Engineering, Faculty of Industrial and Mechanical Engineering, \\ Qazvin Branch, Islamic Azad University, Qazvin, Iran
}

\begin{abstract}
Among all deadly illness, heart outbreaks diseases are considered as the greatest prevailing. Data envelopment analysis (DEA) offers a relative efficiency measure for peer decision-making units (DMUs) with multiple inputs and outputs. Evaluating of hospitals is one of the major applications in DEA. In this study, the input-oriented is used. The window analysis model was presented as a model that is used to estimate the Technical Efficiency (TE), pure technical efficiency (PTE), and Scale Efficiency (SE). The data covers a six-year span from 2011 to 2016 for 12 local heart hospitals. Three inputs (which is two inputs for the first stage and one for the intermediate element of a two-stage model) and two outputs are selected and these factors reveal the main function of hospitals. Finally, actual, target and potential improvement of the smallest and largest TE, PTE, and SE average of the first hospital are compared. The system was implemented in Banxia Frontier Analyst software.
\end{abstract}

Keywords: data envelopment analysis, window analysis, intermediate element, two-stage model, frontier software

\section{Introduction}

Health physicians bearing diverse investigations on heart diseases and gather information of heart patients, their symptoms and disease development. Progressively are reported for patients with common diseases who have typical symptoms. Per annum, 17.3 million people around die from heart disease. Therefore, there is respected information unknown in their dataset to be extracted [1].

The most public used technique, Data Envelopment Analysis (DEA) based on linear programming, appeals a frontier of best practices and shows which health administrations are efficient or inefficient. DEA gives the extent of inefficiency and specifies the means of improving efficiency by giving targets projections for all inputs and outputs independently [2].

To discover the unfamiliar trends in heart disease, all the accessible hospital's datasets are applied to a distinctive model and their DMUs efficiency are compared. A dataset for 12 hospitals with two inputs, one intermediate element and two outputs in a new two-stage model for DEA window analysis, which are $\mathrm{CCR}_{\mathrm{IO}}$ and $\mathrm{BCC}_{\mathrm{IO}}$ to test and validate the alterations between hospitals are used. DEA, as a decision analysis tool, is vast in literature since it does not concentration on discovery a general connection for all units under assessment in the sample. DEA authorities every unit in the data to have its own production function and then it evaluates the efficiency of that single unit by comparing it to the efficiency of the other units in the dataset. After running the DEA model in Banxia Frontier Analyst software with every unit in the data, this study classifies all units into three groups: High efficient (H-efficient) with 100\% efficiency scores, efficient with between $90 \%$ and $100 \%$ efficiency scores, and inefficient with less than $90 \%$ efficiency scores.

\section{Background and literature review}

Universal rising number of patients have inspired researchers to do comprehensive research to reveal unknown patterns in clinical datasets. This section delivers an outline of preceding computational studies on outline recognition in hospitals. Caballer-Tarazona et al (2010) dignified the efficiency of 22 hospitals in the Valencian portion of Spain in expressions of three units [3]. Therefore, pattern recognition in hospitals can be addressed through different computational models. Other respected works, focused on diverse computational aspects of 
hospitals on different models including data mining can be mentioned [4-5-6-7]. Finally, the output-oriented model is used. A unique utilizing window analysis and a novel two-stage model is applied in this study.

\section{Dataset Description}

The standard dataset, collected in this study contains 6 periods (2011-2016), which is collected from 12 hospitals, under the management of National Health Ministry. The Number of Doctors $(\mathrm{NN})$ and the Number of Beds (NB) in the hospitals are inputs in the first stage. The Numbers of Nurses and secretaries (NN) are an intermediate element. The Outpatient Treated (OT) and the Inpatient Treated (IT) in the hospitals are outputs in the second stage. So, two inputs, one intermediate element and two outputs for the first hospital in all periods (20112016) are presented in Table I.

TABLE I. The inputs, intermediate elements, and outputs for 6 DMUs in the first hospitals

\begin{tabular}{llllll}
\hline \hline Period & First input & Second input & $\begin{array}{l}\text { Intermediate } \\
\text { elements }\end{array}$ & First output & Second output \\
\hline & NN (units) & ND (units) & $\begin{array}{l}\text { NB (units) } \\
1450\end{array}$ & $\begin{array}{l}\text { OT (units) } \\
25300\end{array}$ & $\begin{array}{l}\text { IT (units) } \\
5693\end{array}$ \\
\hline 2011 & 16200 & 200 & 6100 & 17200 & 4463 \\
\hline \hline 2012 & 11046 & 158 & 560 & 24100 & 5000 \\
\hline \hline 2013 & 15000 & 260 & 7100 & 16100 & 1800 \\
\hline \hline 2015 & 13601 & 188 & 2450 & 17693 & 4500 \\
\hline \hline 2016 & 14300 & 201 & 482 & 27500 & 9800 \\
\hline \hline
\end{tabular}

\section{Research methodology}

\subsection{The CCR and BCC model}

1) CCR Model: The CCR models reflects a stable or constant return to scale (CRS), which means that a relative increase in all inputs is equal to increase in outputs. The efficiency of a given DMU is considered using the CCR model as follows:

$\operatorname{Min} \theta_{p}$

$$
\begin{array}{ll}
\text { s.t } & \sum_{j=1}^{n} \lambda_{j} x_{i j} \leq \theta_{p} x_{i p} \quad, i=1, \ldots, m \\
& \sum_{j=1}^{n} \lambda_{j} y_{r j} \geq y_{r p} \quad, r=1, \ldots, s \\
\lambda j \geq 0, \quad j=1, \ldots, n &
\end{array}
$$

Where $\theta_{p}$ signifies the technical efficiency score of unit DMU, $\lambda_{j}$ represents the dual variables that identify the benchmarks for inefficient units. If $\theta_{p}$ is set to one, then the examined DMU is considered technically efficient and lies on the efficiency frontier that is composed from the set of efficient units. DEA measures the efficiency of each observation relative to the frontier that envelopes all the observations. Inefficient DMUs can be improved (moved to the efficient frontier) with proposed directions for improvement which are the points along the frontier. The distance to the efficiency frontier provides a measure of the efficiency.

2) BCC Model: On the other hand, the BCC model by Banker-Charles-Cooper transformed the Constant Return to Scale (CRS) concept to Variable Return to Scale (VRS). The DMU controls under variable returns to scale and it is suspected that an increase in inputs does not result in a comparative change in the outputs. The BCC model splits the Technical Efficiency (TE) resulting from the CCR model into two parts: 
- Pure Technical Efficiency (PTE): PTE, which overlooks the influence of scale size by only comparing a DMU to a unit of similar scale and measures how a DMU utilizes its sources under exogenous environment.

- Scale Efficiency (SE): SE, which measures how the scale size affects efficiency. If after applying both CRS, VRS model on the same data, there is an alteration in the two technical efficiencies, this designates that DMU has a scale efficiency and can be calculated by:

$$
S E=T E / P T E
$$

The BCC is represented as follows:

$$
\begin{aligned}
& \operatorname{Min} \theta_{p} \\
& \text { s.t } \sum_{j=1}^{n} \lambda_{j} x_{i j} \leq \theta_{p} x_{i p} \quad, i=1, \ldots, m \\
& \begin{array}{ll}
\sum_{j=1}^{n} \lambda_{j} y_{r j} \geq y_{r p} & , r=1, \ldots, s \\
\sum_{j=1}^{n} \lambda_{j}=1 &
\end{array} \\
& \lambda_{j} \geq 0, j=1, \ldots, n
\end{aligned}
$$

\subsection{A new approach in DEA two-stage model}

In recent years Kao et al., 2008 [8] and Wang et al., 2010 [9] suggested various solutions regarding the twostage model. A new two-stage model has been offered in this study:

In the proposed solution the two-stage model is considered as a single stage one, where the intermediate elements are considered as part of final inputs. Consider the input, intermediate element, and output are denoted by $\mathrm{X}, \mathrm{Z}$, and $\mathrm{Y}$, then the proposed model treats $\mathrm{X}+\mathrm{Z}$, and $\mathrm{Y}$ as input and output, respectively. This model is proposed to regulate the efficiency of a two-stage process, and avoid the reliance to different weights. With respect to aforesaid information, the proposed model is denoted in Figure 1.

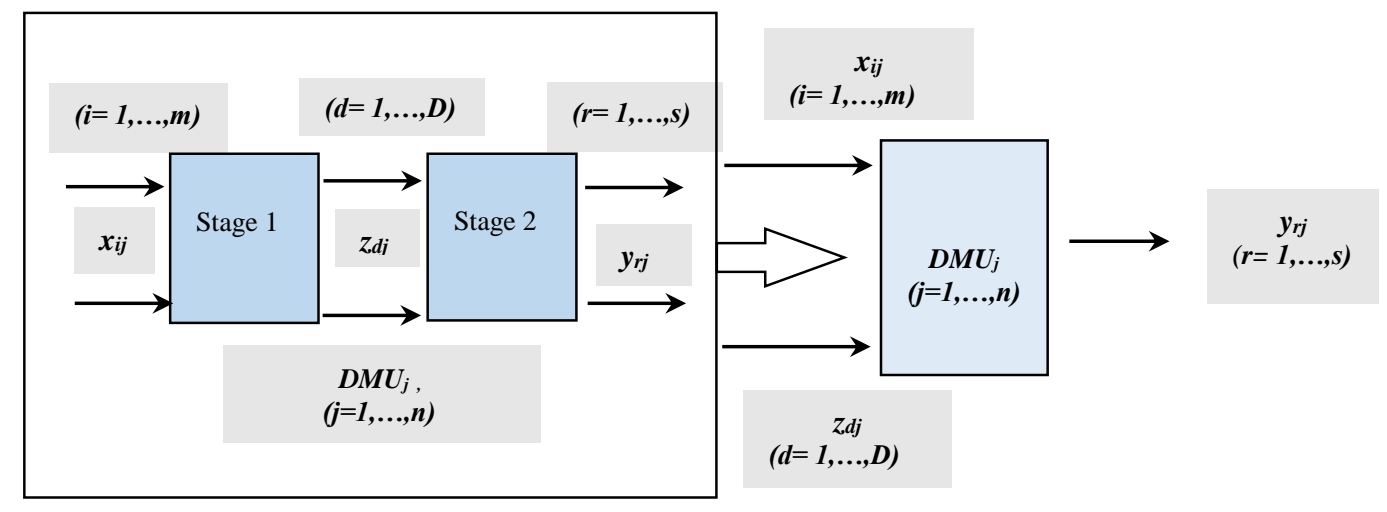

Fig. 1: Conversion of the two-stage model to one-stage model

Figure 1 elaborates the proposed model. In fact, NN with ND units represent $\mathrm{X}$ or inputs, NB unit is Z or intermediate elements and OT with IT units are Y or outputs, which were introduced in table 1. DEA window is a non-parametric panel attitude analysis and it can simplify the concept of moving averages to divulge the efficiency trends of DMUs over time. Each DMU in a window is reflected as a completely different one. DMU's concert in a specific period is contrasted with its performance in other periods in addition to the performance of the other DMUs. Finally, in a more detailed discussion $\mathrm{CCR}_{\mathrm{IO}}$ and $\mathrm{BCC}_{\mathrm{IO}}$ within all approaches, are thoroughly discussed below, at first linear $\mathrm{CCR}_{\mathrm{IO}}$ : 


$$
\begin{aligned}
& \operatorname{Max} \sum_{r=1}^{s} u_{r} y_{r p} \\
& \text { s.t. } \sum_{i=1}^{m} v_{i} x_{i j}+\sum_{d=1}^{D} w_{d} z_{d j}=1 \\
& \sum_{r=1}^{s} u_{r} y_{r j}-\sum_{i=1}^{m} v_{i} x_{i j}-\sum_{d=1}^{D} w_{d} z_{d j} \leq 0 \quad, j=1, \ldots, n \\
& u_{r}, v_{i}, w_{d} \geq \varepsilon
\end{aligned}
$$

Dual proposed model in $\mathrm{CCR}_{\mathrm{IO}}$ :

$$
\begin{aligned}
& \operatorname{Min} \theta_{p} \\
& \text { s.t. } \sum_{j=1}^{n} \lambda_{j} x_{i j} \leq \theta x_{i p} \quad, i=1, \ldots, m \\
& \begin{array}{ll}
\sum_{j=1}^{n} \lambda_{j} y_{r j} \geq y_{r p} & , r=1, \ldots, s \\
\sum_{j=1}^{n} \lambda_{j} z_{d j} \leq \theta z_{d p} & , d=1, \ldots, D
\end{array} \\
& \lambda_{j} \geq 0, \theta_{p} \text { free } j=1, \ldots, n
\end{aligned}
$$

Linear proposed model in $\mathrm{BCC}_{\mathrm{IO}}$ :

$$
\begin{aligned}
& \operatorname{Max} \sum_{r=1}^{s} u_{r} y_{r p}+w \\
& \text { s.t. } \sum_{i=1}^{m} v_{i} x_{i j}+\sum_{d=1}^{D} w_{d} z_{d j}=1 \\
& \sum_{r=1}^{s} u_{r} y_{r j}-\sum_{i=1}^{m} v_{i} x_{i j}-\sum_{d=1}^{D} w_{d} z_{d j}+w \leq 0 \quad, j=1, \ldots, n \\
& u_{r}, v_{i}, w_{d} \geq \varepsilon, w \text { free }
\end{aligned}
$$

Dual proposed model in $\mathrm{BCC}_{\mathrm{IO}}$ :

$\operatorname{Min} \varphi$

$$
\begin{aligned}
& \text { s.t. } \sum_{j=1}^{n} \lambda_{j} x_{i j} \leq \varphi x_{i p} \quad, i=1, \ldots, m \\
& \sum_{j=1}^{n} \lambda_{j} y_{r j} \geq y_{r p} \quad, r=1, \ldots, s \\
& \sum_{j_{\mathfrak{F} 1} 1} \lambda_{j} z_{d j} \leq \theta z_{d p} \quad, d=1, \ldots, D \\
& \sum_{j=1} \lambda_{j}=1 \\
& \lambda_{j} \geq 0, \theta_{p} \text { free } j=1, \ldots, n
\end{aligned}
$$




\subsection{Evaluation in window analysis}

When there is an inadequate number of DMUs, DEA window analysis permits the comparison of a DMUs efficiency in a particular period. To enlarge our comparison, input-oriented standard assessment methods are considered to analyze and ranking each $\mathrm{H}$-efficient, efficient and inefficient DMUs of aforementioned phases.

Figure 2 elaborates the proposed model.

Data with 2 inputs, 1 intermediate elements and 2 outputs for 12 hospitals in 6 periods

A new two-stage model with conversion data to one stage with 3 inputs and 2 outputs

JL

Presenting the average of TE, PTE and SE values, using CCR and BCC models in three windows of all hospitals and finally the highest and lowest performance hospital are introduced
Determining extra inputs and deficiency of efficiency for first hospital and the specific quantity of input that inefficient and efficient DMUs needs to decrease in order to become H-efficient

Fig. 2 Implementation of DEA for efficiency analysis

\section{Result and discussion}

The window analysis reflects each blowing hospital as a dissimilar hospital in each of the halves listed at the top of the table in order to attain the scores registered in the rows that constitute the window, while the stub on the left side shows the window length and the periods covered. For example, the first row extends from 2011 to 2014 for a window length of four splits that is exhibited in the first row. The next row starts in the second year (2012) and extends to 2015 which embodies another window and so on. This results in three windows. Then, there are different data points to which the DEA model is applied to acquire the efficiency scores. Firstly, the Technical Efficiency (TE) and Pure Technical Efficiency (PTE) are computed using CCR and BCC models, separately. The Scale Efficiency (SE) is then calculated. Tables II, III and IV present the TE, PTE and SE values for the first hospital, respectively. To demonstrate, the first hospital in the first window extends from 2011 to 2014 for a window length of 4 years, in 2014, TE $(=0.752)$, PTE $(=0.845)$ and finally SE is calculated as follows: $S E=T E / P T E=0.7520 .845=0.889$

TABLE II. Window analysis for TE in the first hospital

\begin{tabular}{|c|c|c|c|c|c|c|c|c|c|c|c|}
\hline $\mathrm{TE}$ & 2011 & 2012 & 2013 & 2014 & 2015 & 2016 & Avg. & Std. & $\mathrm{CV}$ & Min & Max \\
\hline $2011-2014$ & 1 & 1 & 1 & 0.752 & & & 0.938 & 0.124 & 0.132 & 0.752 & 1 \\
\hline 2012-2015 & & 1 & 1 & 0.789 & 1 & & 0.947 & 0.105 & 0.11 & 0.789 & 1 \\
\hline 2013-2016 & & & 1 & 0.924 & 0.956 & 1 & 0.97 & 0.037 & 0.038 & 0.924 & 1 \\
\hline Avg. & 1 & 1 & 1 & 0.821 & 0.978 & 1 & & & & & \\
\hline \multicolumn{12}{|c|}{ TABLE III. Window analysis for PTE in the first hospital } \\
\hline PTE & 2011 & 2012 & 2013 & 2014 & 2015 & 2016 & Avg. & Std. & $\overline{\mathrm{CV}}$ & Min & Max \\
\hline $2011-2014$ & 1 & 1 & 1 & 0.845 & & & 0.9612 & 0.08 & 0.132 & 0.845 & 1 \\
\hline $2012-2015$ & & 1 & 1 & 0.846 & 1 & & 0.9615 & 0.08 & 0.11 & 0.846 & 1 \\
\hline 2013-2016 & & & 1 & 1 & 1 & 1 & 1 & 0 & 0 & 1 & 1 \\
\hline Avg. & 1 & 1 & 1 & 0.897 & 1 & 1 & & & & & \\
\hline \multicolumn{12}{|c|}{ TABLE IV. Window analysis for SE in the first hospital } \\
\hline SE & 2011 & 2012 & 2013 & 2014 & 2015 & 2016 & Avg. & Std. & $\overline{\mathrm{CCV}}$ & Min & Max \\
\hline 2011-2014 & 1 & 1 & 1 & 0.889 & & & 0.974 & 0.05 & 0.051 & 0.899 & 1 \\
\hline 2012-2015 & & 1 & 1 & 0.932 & 1 & & 0.983 & 0.034 & 0.035 & 0.932 & 1 \\
\hline 2013-2016 & & & 1 & 0.924 & 0.956 & 1 & 0.97 & 0.037 & 0.038 & 0.924 & 1 \\
\hline Avg. & 1 & 1 & 1 & 0.918 & 0.978 & 1 & & & & & \\
\hline
\end{tabular}

Applying the aforementioned classification of H-efficient (100\%), Efficient (between 90\% and 100\%) and Inefficient (less than 90\%) means that all three windows in TE are Efficient. The first two windows of PTE are Efficient and the last window of PTE is H-efficient. All three windows in SE are Efficient. The columns and rows of window analysis offer two distinct determinations: 
- $\quad$ The columns in window DEA are used to survey permanency possessions. The TE, PTE and SE values of the first hospital in Table II showed a stable performance for two periods. Actually, TE, PTE, and SE in 2012 and 2013 are set to 1 for three windows.

- The row window is examined to classify the performance trends. For example in the first row, the TE average is 0.938 and a standard deviation is set to 0.124 . It is found that the Coefficient of Variation (CV) for TE values in all windows apart from the last window for the first hospital are larger than 5\%, which shows the existence of trends in the efficiencies of the same window.

The results in Table V, VI, VII, and VIII are used for determining extra inputs and deficiency of efficiency for the first hospital and the specific quantity of input that inefficient and efficient DMUs needs to decrease in order to become H-efficient. In fact, actual, target and potential enhancement of DMUs which are not $\mathrm{H}$-efficient in each window, are introduced in order to stand on the efficiency frontier line for the smallest and largest TE and PTE average values. Unless largest PTE average, which is H-efficient in that actual is set with the target and potential improvement, other averages need to decrease the number of inputs.

Table V. The only inefficient DMU (0.752) in 2014 in the smallest TE Avg. (0.938)

\begin{tabular}{llcl}
\hline \hline Variable & Actual & Target & Improvement \\
\hline Input1(NN) & 13601 & 10222 & $-24.84 \%$ \\
Input2(ND) & 188 & 142 & $-24.84 \%$ \\
Input3(NB) & 7100 & 757 & $-89.34 \%$ \\
\hline \hline
\end{tabular}

Table VI. The first efficient DMU (0.924) in 2014 in the largest TE Avg. (0.97)

\begin{tabular}{llcl}
\hline \hline Variable & Actual & Target & Improvement \\
\hline Input1(NN) & 13601 & 10222 & $-24.84 \%$ \\
Input2(ND) & 188 & 174 & $-7.61 \%$ \\
Input3(NB) & 7100 & 374 & $-94.73 \%$ \\
\hline \hline
\end{tabular}

Table VII. The second efficient DMU (0.956) in 2015 in the largest TE Avg. (0.97)

\begin{tabular}{llrl}
\hline \hline Variable & Actual & Target & Improvement \\
\hline Input1(NN) & 14300 & 9996 & $-30 \%$ \\
Input2(ND) & 201 & 192 & $-4.4 \%$ \\
Input3(NB) & 2450 & 379 & $-84.52 \%$ \\
\hline \hline
\end{tabular}

Table VIII. The only inefficient DMU (0.845) in 2014 in the smallest PTE Avg. (0.9612)

\begin{tabular}{llcl}
\hline \hline Variable & Actual & Target & Improvement \\
\hline Input1(NN) & 13601 & 11156 & $-17.98 \%$ \\
Input2(ND) & 188 & 159 & $-15.48 \%$ \\
Input3(NB) & 7100 & 6001 & $-15.48 \%$ \\
\hline \hline
\end{tabular}

From Tables II, III, IV, V, VI, VII, and VIII the following results are obtained:

The first window of TE for the first hospital (2011-2014) produced the smallest TE average value $(=0.938)$. The TE for the only DMU which is not H-efficient in 2014 (=0.752) infers that the same level of output could be produced with $75.2 \%$ of the resources if this DMU was performing on the Frontier. It could be interpreted also that $24.08 \%$ of overall resources could be saved by raising the performance of this DMU to the highest level. In Table V, in order for the first hospital in this DMU to become H-efficient, it needs to decrease 3379 or $24.84 \%$ units of the NN and decrease 46 or $24.84 \%$ of ND in addition to decrease 6343 units of the NB. On the one hand, other DMUs of this window stands on the efficiency frontier line and does not need to decrease any of the inputs. All aforementioned steps can be inferred from the largest TE and the smallest and the largest PTE. Table IX displays a classification of TE, PTE, and SE efficiency values in each window for all hospitals. 
TABLE IX. Average efficiency of each window for TE, PTE, and SE in all hospitals

\begin{tabular}{|c|c|c|c|c|c|c|c|c|c|c|c|}
\hline $1^{\mathrm{ST}}$ hospital & $\mathrm{TE}$ & PTE & SE & $2^{\text {nd }}$ hospital & $\mathrm{TE}$ & PTE & SE & $3^{\text {rd }}$ hospital & $\mathrm{TE}$ & PTE & SE \\
\hline $2011-2014$ & 0.938 & 0.961 & 0.974 & $2011-2014$ & 0.864 & 0.866 & 0.997 & $2011-2014$ & 0.949 & 0.991 & 0.958 \\
\hline $2012-2015$ & 0.947 & 0.961 & 0.983 & $2012-2015$ & 0.866 & 0.894 & 0.967 & 2012-2015 & 0.918 & 0.988 & 0.929 \\
\hline 2013-2016 & 0.970 & 1 & 0.970 & 2013-2016 & 0.926 & 0.935 & 0.989 & 2013-2016 & 0.958 & 1 & 0.958 \\
\hline $4^{\text {th }}$ hospital & TE & PTE & SE & $5^{\text {th }}$ hospital & TE & PTE & SE & $6^{\text {rd }}$ hospital & TE & PTE & SE \\
\hline 2011-2014 & 0.768 & 0.796 & 0.964 & 2011-2014 & 0.639 & 0.809 & 0.789 & 2011-2014 & 0.966 & 0.971 & 0.994 \\
\hline $2012-2015$ & 0.856 & 0.899 & 0.951 & $2012-2015$ & 0.717 & 0.863 & 0.830 & $2012-2015$ & 0.931 & 0.943 & 0.987 \\
\hline $7^{\text {th }}$ hospital & $\mathrm{TE}$ & PTE & SE & $8^{\text {th }}$ hospital & TE & PTE & SE & $9^{\text {th }}$ hospital & $\mathrm{TE}$ & PTE & SE \\
\hline $2011-2014$ & 1 & 1 & 1 & $2011-2014$ & 0.999 & 1 & 0.999 & $2011-2014$ & 1 & 1 & 1 \\
\hline $2012-2015$ & 1 & 1 & 1 & $2012-2015$ & 1 & 1 & 1 & $2012-2015$ & 1 & 1 & 1 \\
\hline 2013-2016 & 1 & 1 & 1 & 2013-2016 & 0.916 & 1 & 0.916 & 2013-2016 & 0.919 & 0.982 & 0.936 \\
\hline $10^{\text {th }}$ hospital & TE & PTE & SE & $11^{\text {th }}$ hospital & TE & PTE & SE & $12^{\text {th }}$ hospital & TE & PTE & SE \\
\hline
\end{tabular}

Decomposing technical efficiency scores into PTE and SE distributes regulation on what can be accomplished in the short and long associations. If the majority of inefficiency in any hospital is due to the low importance of SE which means a small size of medicinal operation, then the hospital needs to be extended. Conversely, the PTE value can be usually addressed in the short term without altering the scale of medical operations. The low value of PTE is due to managerial deficit problem in organizing the inputs in the medical process. It should be distinguished that it is easier to increase the TE than to increase SE. When a hospital becomes technically efficient, that makes sense to deal with SE, so hospital administrator should focus on increasing the TE. For example, in Table IX, the TE for the fifth hospital in the first window (2011-2014) with 0.639 is considered inefficient because of low SE (= 0.789). PTE in this window is 0.809 . Then, the low TE value in this window is attributed to the low performance in scale efficiency and the development is the solution in this case. Additionally, the TE for the fourth hospital in the first window of 0.768 is inefficient because of low PTE (=0.796). SE in this window is 0.964. Then, the low TE value in this window is attributed to the low performance in PTE due to low utilization of the inputs or a problem in transforming inputs into outputs and the number the outpatient treated (OT) and inpatient treated (IT) in the hospitals should be increased. The efficiency categories and an average efficiency of each window for TE, PTE, and SE for all hospitals, corresponds to each category are considered and finally, Seventh hospital with H-efficient category in all windows of TE, PTE, and SE is considered as the highest performance hospital. The fifth hospital with the inefficient category in all windows of TE, PTE, and SE is considered as the lowest performance hospital.

\section{Conclusion}

Several heart hospitals efficiencies in DEA were compared. A unique model involving of an exclusive model and transforming two-stage model to a novel one stage model and evaluation methods are evolved. Input-oriented window analysis with TE, PTE, and SE are applied to find the superior hospitals and periods. Hospitals are compared in terms of their actual, target and potential improvement. The highest and lowest performance hospitals within various angle were introduced. The experimentation can assist as a practical instrument for the general practitioner to effectively compare the efficiency of uncertain cases and instruct accordingly.

\section{References}

[1] S. Mendis, P. Puska, B. Norring, "World Health Organization Global Atlas on Cardiovascular Disease Prevention and Control," 2011. S. Mendis, P. Puska, B. Norring, "World Health Organization Global Atlas on Cardiovascular Disease Prevention and Control," 2011.

[2] M.S.C Lobo, Y Ozcan, M.P.E Lins, A.C.M Silva, R. Fiszman, "Financing reform and productivity change in Brazilian teaching hospitals: Malmquist approach,” Central European Journal of Operations Research 18:141-152, 2010. 
[3] M. Caballer-Tarazona, I. Moya-Clemente, D. Vivas-Consuelo and I. Barrachina-Martínez "A model to measure the efficiency of hospital performance," Mathematical and Computer Modelling 52: 1095-1102, 2010.

[4] M. Mirmozaffari, A. Alinezhad, and A. Gilanpour, "Data Mining Classification Algorithms for Heart Disease Prediction," Int'l Journal of Computing, Communications \& Instrumentation Engg (IJCCIE), ISSN 2349-1469 EISSN 2349-1477, Vol.4, Issue1, Jan 2017.

[5] M. Mirmozaffari, A. Alinezhad, and A. Gilanpour, "Heart Disease Prediction with Data Mining Clustering Algorithms," Int'l Journal of Computing, Communications \& Instrumentation Engg (IJCCIE), ISSN 2349-1469 EISSN 2349-1477, Vol.4, Issue1, Jan 2017.

[6] M. Mirmozaffari, A. Alinezhad, and A. Gilanpour, "Data Mining Apriori Algorithm for Heart Disease Prediction," Int'l Journal of Computing, Communications \& Instrumentation Engg (IJCCIE), ISSN 2349-1469 EISSN 2349-1477, Vol.4, Issue 1, Jan 2017.

[7] M. Mirmozaffari, A. Alinezhad. "Ranking of Heart Hospitals Using Cross-Efficiency and Two-stage DEA," 7th International Conference on Computer and Knowledge Engineering (ICCKE 2017), October 26-27 2017, Ferdowsi University of Mashhad, 978-1-5386-0804-3/17/\$31.00 @2017 IEEE.

[8] C. Kao and S.N. Hwang, (2008), Efficiency Decomposition in Two-Stage Data Envelopment Analysis: an Application to non-Life Insurance Companies in Taiwan. European Journal of Operational Research 185, 418-429.

[9] Y.M. Wang, and K.S. Chin, (2010) Some Alternative DEA Models for Two-stage Process. Expert Systems with Applications 37, 8799-8808. 